\title{
Evaluaciones económicas en el proceso de toma de decisiones en salud: El caso de Inglaterra
}

\author{
CARLA CASTILLO-LABORDE ${ }^{1, a}$
}

\section{Economic evaluation in the health care decision making process: The case of England}

Economic evaluation plays an increasingly important role in the health decision making process. Many countries, mainly developed ones, have incorporated these kinds of methodologies in their priority setting processes. The creation of NICE in England is one of the clearer examples of how economic evaluations can be incorporated in a systematic, structured and explicit way, having a strong influence on what is or what is not finally funding by the National health Service (NHS). Although in Latin America the development and use of economic evaluations is neither massive nor systematic, there have been significant advances in recent years. Examples of this are the cases of Mexico and Chile. These two countries have incorporated economic criteria in the priority setting process and the selection of interventions in the context of their health reforms. Alternatives to go further in the incorporation of these kinds of approaches in Latin American countries are the generalizability and transferability. Even though these options must be considered with caution, they allow to apply in different settings the results of studies developed in specific settings.

(Rev Med Chile 2010; 138 (Supl 2): 103-107).

Key words: Economic evaluation, decision making, UK, Chile, transferability.

\section{RESUMEN}

Las evaluaciones económicas juegan un rol cada vez más importante en el proceso de toma de decisiones en salud, muchos países, especialmente países desarrollados, han incorporado este tipo de metodologías en sus procesos de priorización. La creación del NICE en Inglaterra es uno de los ejemplos más ilustrativos de cómo las evaluaciones económicas pueden ser incorporadas de manera sistemática, estructurada y explicita, teniendo finalmente una fuerte injerencia en lo que resulta o no financiado por el Servicio Nacional de Salud (NHS). Si bien en América Latina el desarrollo y uso de las evaluaciones económicas no es aún masivo ni sistemático, en los últimos años se ha avanzado en su incorporación. Un ejemplo de esto son los casos de México y Chile y la inclusión de criterios económicos en el proceso de priorización y selección de las intervenciones en el contexto de la reforma de salud. Una alternativa para avanzar en la incorporación de dichas evaluaciones en países latinoamericanos, que debe ser considerada con precaución, está dada por la generalización y transferibilidad, que permite de estudios realizados en contextos especificos, puedan ser aplicados a realidades distintas.

Palabras clave: Evaluación económica, toma de decisiones, UK, Chile, transferabilidad.

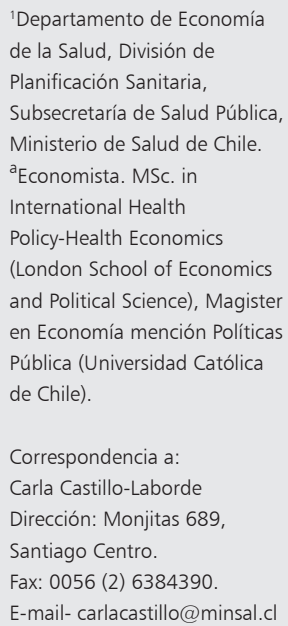

'Departamento de Economía de la Salud, División de Planificación Sanitaria, Subsecretaría de Salud Pública, Ministerio de Salud de Chile. aEconomista. MSc. in International Health Policy-Health Economics (London School of Economics and Political Science), Magister en Economía mención Políticas Pública (Universidad Católica de Chile).

Correspondencia a: Carla Castillo-Laborde Dirección: Monjitas 689, Santiago Centro. Fax: 0056 (2) 6384390 E-mail-carlacastillo@minsal.cl 
L as evaluaciones económicas, prácticas esporádicas en el contexto chileno de toma de decisiones en salud, son parte del día a día en otros países, siendo Inglaterra uno de los más claros ejemplos.

EL NICE o Instituto Nacional para Salud y Excelencia Clínica fue creado en 1999 bajo el alero del Servicio Nacional de Salud inglés o NHS, como una forma de mejorar los estándares de atención de pacientes, al mismo tiempo de evitar el llamado 'post-code lottery' (variaciones en la atención de salud recibida dependiendo del distrito en que correspondía atenderse) y por lo tanto, lograr un sistema de salud más igualitario. A partir de la evaluación en términos de eficacia y costo-efectividad de tratamientos y fármacos, el NICE entrega recomendaciones o guías respecto de qué tecnologías son las convenientes de cubrir por parte de NHS.

En el caso de los países de Latino America, el desarrollo y uso de las evaluaciones económicas está muy por detrás del de países como Inglaterra. La motivación del presente artículo es presentar en cierto grado de detalle el proceso de formulación de guías clínicas por parte del NICE, además de discutir a partir de la literatura aspectos relevantes del proceso de toma de decisiones en salud en Inglaterra. A partir de esto, se establece un contraste con el uso de evaluaciones económicas en América Latina, especialmente en el caso de Chile, y las potencialidades de su incorporación más masiva. En este contexto, también se discute el uso de la generalización y transferibilidad.

\section{Evaluaciones económicas en el proceso de toma de decisiones en salud: Inglaterra}

El NHS es financiado públicamente sobre la base de impuestos, cubriendo de esta forma a la totalidad de los residentes en el país. Es así como la inclusión del NICE en el proceso de toma de decisiones afectaría al menos dos niveles de equidad. La primera es igual acceso independiente de la capacidad de pago de los residentes, esto en el sentido de que toda tecnología recomendada por el NICE será financiada y por lo tanto, generalmente gratis en el punto de atención. La segunda es igual acceso independiente de la zona en que las personas vivan, evitando el antes mencionado 'post-code lottery'.
El NICE está estructurado en centros y directorios. Mientras los directorios proveen soporte a los centros y otras actividades corporativas, los centros son los encargados de realizar las guías. El Instituto es responsable de proveer guías nacionales en tres áreas de salud, cada área bajo la responsabilidad de uno de los 3 centros de excelencia: Centro para la Excelencia en Salud Pública (promoción y prevención de enfermedad), Centro para Evaluación de Tecnologías de Salud (guías sobre el uso tratamientos, procedimientos y medicinas ya sean nuevos o existentes), Centro para Práctica Clínica (guías sobre tratamientos y cuidados de personas con enfermedades específicas).

El proceso a partir del cual el NICE finalmente recomendará o no una tecnología es complejo y considera distintos actores. Las guías NICE son desarrolladas por grupos consultores independientes, conformados por profesionales de la salud, trabajadores de NHS, pacientes y sus cuidadores y por el público. Además, el proceso está estructurado en una serie de pasos ${ }^{1}$.

En primer lugar, el Departamento de Salud indica un tópico de interés al NICE. Luego son identificados los distintos grupos interesados y afectados con el tema (organizaciones de pacientes, cuidadores, cuerpos de profesionales), los cuales son consultados e integrados a lo largo del proceso. Considerando lo anterior, el centro encargado de elaborar la guía debe preparar el alcance de ésta (qué será cubierto y qué no). Además, se establece un grupo de desarrollo de la guía (profesionales de la salud, representantes de grupos de interés, expertos), el cual a partir de la revisión de la evidencia disponible, produce un borrador de ésta.

Existe una instancia para que los interesados (registrados) comenten el borrador de la guía; además, existe un revisor independiente, de manera de asegurar que estos comentarios hayan sido incluidos. Una vez que el grupo de desarrollo de la guía termina con las recomendaciones, el centro de colaboración encargado produce la guía final. NICE finalmente aprueba la guía, pasando ésta a formar parte de las recomendaciones para el NHS.

Uno de los hitos que fortaleció de manera importante el rol del NICE es la declaración como obligatorias de sus decisiones por parte del NHS en Inglaterra y Gales en el año $2002^{2}$. De esta forma, las decisiones de cobertura se toman a nivel centralizado, mientras que sólo la implementación queda en manos locales. 
Una pregunta que surge inevitablemente es: ¿cuáles son los factores que influyen en la recomendación o no por parte del NICE de una tecnología en evaluación? Una hipótesis ampliamente difundida, aunque nunca claramente explicitada o aceptada por el NICE, es la existencia de un umbral de costo efectividad por sobre el cual una tecnología no resultaría recomendada por la institución.

Devlin y Parkin ${ }^{2}$, exploraron la existencia de este umbral, sugiriendo que el 'rango de costo efectividad aceptable' se encontraría entre $\mathfrak{E} 35.000$ y $\mathfrak{E} 40.000$ per QALY. En otras palabras, tecnologías con una razón de costo efectividad mayor que este rango tienen mayor probabilidad de ser rechazadas (artículos anteriores a éste han señalado que el um-

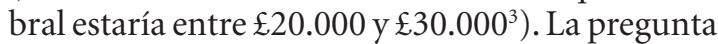
que sigue es si este umbral es categórico y por lo tanto, cada vez que la razón costo efectividad de una tecnología excede el umbral, ésta es rechazada, y si cae dentro, es aceptada; o si existen otros factores que influyen en la decisión, generando una especie de trade-off. En este sentido, los autores también encontraron evidencia de que la razón costo efectividad no es el único factor considerado en la toma de decisiones, otros factores considerados serían también la carga de enfermedad y la incertidumbre en cuanto a la evidencia disponible. En esta revisión Davlin y Parkin ${ }^{2}$ encontraron una gran heterogeneidad en los datos usados en el análisis, con distintas medidas de outcomes (años de vida ganados, QALYs) y de carga de enfermedad (casos, caso nuevos, tratamientos y muertes) y de impacto presupuestario (costos incrementales, costos totales).

Finalmente, otra pregunta que puede surgir a partir de este proceso es: qué impacto tiene, en la práctica, la recomendación o no de una tecnología por parte del NICE? En este contexto, Sheldon et $\mathrm{al}^{4}$, a través del análisis de series de tiempo, la auditoria de fichas de pacientes y entrevistas, evaluaron la extensión y el patrón de implementación de las guías NICE. El estudio concluye que la implementación de dichas guías ha sido variable, dependiendo, entre otras cosas, del apoyo profesional existente en los establecimientos, lo convincente y estable de la evidencia base, si incrementa los costos o no y si este aumento es financiado, etc. En este contexto, existiría evidencia de que tecnologías relacionadas con la prescripción de medicamentos (ej. uso de Taxanos para cáncer de ovario y mamas) se incorporarían de manera más rápida, mientras que las guías relacionadas con procedimientos quirúrgicos serían menos influyentes, al menos en el corto plazo (ej. guía de prótesis de caderas). En cualquier caso, tal como los mismos autores comentan, el estudio es observacional y no existe un contrafactual, por lo que considerando además que hay otros factores que influyen en la práctica clínica, los resultados deben ser analizados con cautela.

En resumen, Inglaterra ha establecido en la última década una política de inclusión explicita y estructurada de las evaluaciones económicas en el proceso de toma de decisiones en salud. El objetivo no sólo es la eficiencia (reducir costos para un objetivo específico, o maximizar las ganancias en salud con los recursos disponibles), a través de la recomendación de las tecnologías más costo efectivas, sino además fomentar la equidad a partir de disminuir la variabilidad de la práctica clínica. La evidencia muestra que la razón de costo efectividad puede no ser la única variable a considerar al momento de recomendar una tecnología, y más aún que a pesar de ser éstas obligatorias, no necesariamente son implementadas con 100\% de cumplimiento por parte de los establecimientos. Sin embargo, esta política corresponde a un esfuerzo pionero y transparente, que en el corto plazo ya ha demostrado buenos resultados y que en el largo plazo podría tener un impacto aún mayor en el manejo de la salud de este país.

\section{Evaluaciones económicas en el proceso de toma de decisiones en salud: América Latina}

Es un hecho que el uso y también la aceptación de las evaluaciones económicas ha sido más masivo en países desarrollados que en países en desarro1lo. En el caso de América Latina, Pichon-Riviere et $\mathrm{al}^{5}$. muestran que los países Latinoamericanos presentan un bajo uso de la evidencia entregada por las evaluaciones de tecnologías sanitarias, poca disponibilidad de recursos para su desarrollo y bajo apoyo del los gobiernos a estas iniciativas. A pesar de esto, países como Brasil, México, Chile y Argentina se encuentran hoy institucionalizando activamente la evaluación de tecnologías sanitarias y ésta también parecería ser la tendencia en otros países del continente ${ }^{6}$.

En México, el año 2003 la reforma de salud crea el Sistema de Protección Social en Salud, 
cuyo componente clave es el Seguro Popular de salud, que a partir de los aportes de los gobiernos (nacional y locales) y de los beneficiarios, financia un sistema explicito de intervenciones en salud. En el proceso de priorización y selección de las intervenciones incluidas en los distintos paquetes (salud pública e intervenciones comunitarias, baja y mediana complejidad o intervenciones esenciales, y de alta complejidad), la carga de enfermedad y las evaluaciones de costo efectividad jugaron un rol fundamental ${ }^{7}$.

El desarrollo de evaluaciones económicas es altamente demandante de información. Sin embargo, información confiable y específica a la realidad local, en términos de la epidemiología, los costos y la efectividad de las intervenciones, no siempre está disponible. Ésta es precisamente una de las principales limitaciones que enfrentan los países en desarrollo al momento de emprender un estudio de esta naturaleza.

La 'generalización' y la 'transferibilidad' ('generalizability' $y$ 'transferability'), surgen entonces como una alternativa ante esta dificultad, como una forma de beneficiarse de la experiencia acumulada y de aquello que ya ha sido estudiado en un contexto distinto, con mejor información disponible. Augustovski et al [p. 921] ${ }^{8}$, definen 'generalización' como 'la medida en que los resultados de un estudio realizado en una población de pacientes particulares y/o contexto específico se mantienen verdaderos para otra población y/o en otro contexto diferente y sin ajustes', $y$ 'transferibilidad', como 'el potencial del análisis de ser ajustado de manera de hacer relevantes sus resultados en un contexto diferente'.

A pesar de los potenciales beneficios, como las evaluaciones económicas son especificas al contexto, no necesariamente son fácilmente generalizables desde un contexto a otro. González-Pier et al. ${ }^{7}$ establecen que a partir de la disponibilidad de información epidemiológica y económica en México, se ha podido constatar las dificultades de transferir conclusiones desde un contexto a otro. Por ejemplo, basados en información epidemiológica local, la vacuna contra la influenza aparece mucho menos costo-efectiva que en estudios para otros países. En el caso del cáncer de mamas, que en México muestra una incidencia mayor en población en edades más joven, una política agresiva de screening resulta costo-efectiva, contrario a lo que sucede por ejemplo en Estados Unidos.
Por otra parte, en su revisión sistemática de evaluaciones económicas que incluyeran al menos un país de América Latina y el Caribe, Augustovski et $\mathrm{al}^{8}$. señalan que algunas barreras asociadas a la generalización y transferibilidad de dichas evaluaciones tienen relación con las deficiencias en el reporte de las características del estudio (pregunta a responder, intervenciones a evaluar, etc) y también en la metodología usada (muchas veces alejadas de los estándares internacionales). Los autores indican que el mejoramiento de estos aspectos es importante a la hora de maximizar los beneficios de la generalización y transferibilidad.

En particular, existe una gran diferencia en el uso y desarrollo de evaluaciones económicas entre países como Inglaterra y Chile. Si bien es cierto, estos dos casos no son comparables directamente, al ser distinta la naturaleza de sus sistemas de salud. Por una parte, Inglaterra cuenta con un sistema público financiado en base a impuestos. Por otra parte, Chile cuenta con un sistema mixto públicoprivado, en el que el seguro público (FONASA) es financiado sobre la base de la seguridad social en salud y aportes fiscales, cubriendo alrededor de $70 \%$ de la población país y un sistema privado (ISAPRE) que cubre a $16 \%$ de la población 9 . En este sentido el NHS cuenta con una ventaja al momento de tomar decisiones centralizadamente. Sin embargo, el FONASA cubre a más de 70\% de la población, dejando por tanto un amplio espacio y oportunidades para la toma de decisiones a nivel central.

La incorporación de este tipo de evaluaciones en el proceso de toma de decisiones asociado tanto a las GES como al quehacer general de los establecimientos de salud en Chile, en conjunto con un carácter obligatorio de las recomendaciones derivadas de éstas, podría resultar en un importante aporte al mejor uso de los recursos escasos y a mejorar los resultados en salud.

\section{Referencias}

1. National Institute for Health and Clinical Excellence. [http://www.nice.org.uk/]. [Acceso 16 de Noviembre de 2009].

2. Devlin N, Parkin D. 'Does NICE have a cost effectiveness threshold and what other factors influence its decisions? A discrete choice analysis. Health Economics 2004; 13 (5): 437-52. 
3. Twose (2002). 'What is NICE's threshold? An external view. Capítulo 2 en: DEVLIN, TOWSE, A. (eds) Cost effectiveness thresholds: economic and ethical issues. 2002 London: King's Fund/Office for Health Economics.

4. Sheldon T, et al. 'What's the evidence that NICE guidance has been implemented? Results from a national evaluation using time series analysis, audit of patients' notes, and interviews'. BMJ 2004; 329 (7473): 999 [doi: 10.1136/bmj.329.7473.999].

5. Pichon-Riviere A, et al. 'HTA in Latin-America and the Caribbean (LAC), facilitators and barriers for international collaboration: a survey'. V Annual Meeting, 9 de Julio, 2008, Montréal Canadá.

6. Banta D. 'Health technology assessment in Latin Ameri- ca and the Caribbean'. International Journal of Technology Assessment in Health Care 2009; 25: 253-2.

7. González-Pier E, et al. 'Priority setting for health interventions in Mexico's System of Social Protection in Health'. Lancet 2006; 368: 1608-18.

8. Augustovski F, et al. 'Barriers to Generalizability of Health Economic Evaluations in Latin America and the Caribbean Region'. Pharmacoeconomics 2009; 27 (11): 919-29.

9. Superintendencia de Salud. Departamento Planeamiento Institucional-Estudios. [http://www.fonasa.cl/ prontus_fonasa/site/artic/20070112/asocfile/01_demografia_pagina_web__08_06_2009_jav.xls\#T1.1.1!A1]. [Acceso 17 de Noviembre de 2009]. 